\title{
Vectorcardiographic ST deviations related to increased heart rate in the absence of ischemia in an experimental pig model
}

\author{
Sören Häggmark ${ }^{\mathrm{a}, *}$, Michael F. Haney ${ }^{\mathrm{b}}$, Göran Johansson ${ }^{\mathrm{b}}$, Björn Biber ${ }^{\mathrm{b}}$, Ulf Näslund ${ }^{\mathrm{c}, \mathrm{d}}$ \\ ${ }^{\mathrm{a} C a r d i o t h o r a c i c ~ S u r g e r y, ~ H e a r t ~ C e n t r e, ~ U n i v e r s i t y ~ H o s p i t a l, ~ U m e a ̊, ~ S E-901 ~} 85$ Umeå, Sweden \\ ${ }^{\mathrm{b}}$ Department of Surgical and Perioperative Science, Anesthesiology and Intensive Care Medicine, Umeå University, Umeå, Sweden \\ ${ }^{\mathrm{c}}$ Cardiology, Heart Centre, University Hospital, Umeå, SE-901 85 Umeå, Sweden \\ ${ }^{\mathrm{d}}$ Department of Public Health and Clinical Medicine, Umeå University, Umeå, SE-901 85 Umeå, Sweden
}

Received 20 June 2005

\begin{abstract}
The electrocardiographic ST segment may change when heart rate (HR) increases. We aimed to analyze vectorcardiographic ST relation and myocardial conditions during controlled HR increases in anesthetized pigs. The relative parameters ST change vector magnitude and ST change vector angle were calculated at paced HRs ranging from 85 to 175 beats per minute. ST change vector magnitude increased from baseline $6.3 \pm 1.3$ to $26.0 \pm 3.1 \mu \mathrm{V}(P<.01$; range, $4-50 \mu \mathrm{V})$ at HR 175 beats per minute with similar changes in ST change vector angle, whereas the absolute parameter ST vector magnitude demonstrated a heterogeneous pattern without any systematic relation to HR changes. Microdialysis results from left ventricular wall, with analysis of glucose, lactate, and pyruvate, showed no sign of ischemia during pacing. Potassium concentrations did not change during pacing. We conclude that significant HR-related ST vector changes can occur in the absence of myocardial ischemia.

(C) 2006 Elsevier Inc. All rights reserved.
\end{abstract}

\section{Introduction}

Electrocardiography (ECG), in particular, ST-segment assessment, is the fundamental method to detect myocardial ischemia and coronary artery disease $[1,2]$. Vectorcardiography is one electrocardiographic method that can be used in diagnostic situations to detect myocardial ischemia and infarction. The ST segment is affected by heart rate (HR), and at higher HRs, ST changes are thought to be less specific for ischemia than at lower HRs $[3,4]$. This negatively affects the sensitivity and specificity of ST analysis for identification of ischemia when higher HRs are present. In a previous study, computerized vectorcardiographic (VCG) ST signals were analyzed during pacing-induced HR increases in patients without coronary artery disease [5]. ST deviations were observed at levels above the voltage threshold that is commonly used for identification of ischemia, although there was

* Corresponding author. Tel.: +46 907850000 .

E-mail address: soren.haggmark@vll.se (S. Häggmark). no definitive metabolic measurement that confirmed absence of ischemia.

Vectorcardiographic ST deviations in relation to HR have not been systematically established, and no work has been published where the presence or absence of ischemic conditions has been simultaneously examined with a second reference method. There are published data for the 12-lead ECG that indicate that ST-segment change has an independent relation to HR, although, again, ischemic conditions have not been absolutely refuted in these studies [6,7]. There is a theoretical possibility that physiological potassium $\left(\mathrm{K}^{+}\right)$ transmembrane flux and increased extracellular $\mathrm{K}^{+}$may be involved in ST deviations with higher HR. We hypothesized that VCG ST change occurs systematically during increasing HR in the absence of ischemia. The aim of the study was to measure ST vector changes during experimental increases in HR in a pig model where there was simultaneous direct and invasive metabolic assessment of myocardial conditions, allowing detection of ischemia if it occurred. Metabolic assessment and analyses of intramyocardial $\left[\mathrm{K}^{+}\right]$were used 
with the aim of identifying possible mechanisms or contributing factors for ST change. Because of the theoretical concern that sternotomy and pericardotomy could diminish the magnitude of electrophysiologic signals from the heart, a second and subsequent aim was to test for possible effect of sternotomy on VCG ST signals in the same HR conditions.

\section{Methods}

\subsection{Materials and preparation}

With approval of the Umeå Research Ethics Committee and in conformity with the Guide for Care and Use of Laboratory Animals [8], 13 female Yorkshire-Hampshire pigs (range, $36-45 \mathrm{~kg}$ ) were used in this study. There were 2 separate groups of animals, which were included in the same pacing protocol. The first group, 9 animals, underwent sternotomy with direct instrumentation of the heart wall. The second group, 4 animals, had no sternotomy.

The animals were premedicated using ketamine (Ketalar; Pfizer, Morris Plains, NJ) $10 \mathrm{mg} / \mathrm{kg}$, azaperone (Stresnil; Janssen-Cilag, Neuss, Germany) $5 \mathrm{mg} / \mathrm{kg}$, and atropine (NM Pharma, Stockholm, Sweden) $0.05 \mathrm{mg} / \mathrm{kg}$ by intramuscular injection. Anesthesia was induced with pentobarbital (Pentobarbitalnatrium; Apoteksbolaget, Stockholm, Sweden) injection $10 \mathrm{mg} / \mathrm{kg}$ intravenous followed by infusions of fentanyl (Fentanyl; Braun, Melsungen, Germany) $20 \mu \mathrm{g} / \mathrm{kg}$ per hour, midazolam (Dormicum; Roche, Basel, Switzerland) $0.3 \mathrm{mg} / \mathrm{kg}$ per hour, and pentobarbital $5 \mathrm{mg} / \mathrm{kg}$ per hour, with the pigs in the supine position. A tracheostomy (7.0 endotracheal tube; Rusch, Kernen, Germany) was placed, and the animals were ventilated (Servo 900B; Siemens, Solna, Sweden) to achieve normoxia and normocapnia. Ringer's acetate was infused first at $25 \mathrm{~mL} / \mathrm{kg}$ for 1 hour and then at $15 \mathrm{~mL} / \mathrm{kg}$ per hour for maintaining normovolemia. Body temperature was kept at approximately $38^{\circ} \mathrm{C}$ with the help of an electronic warming blanket.

\subsection{Catheterization and instrumentation}

All vascular catheterizations were conducted by cut down to vessels in the neck. A 3F Teflon arterial catheter and a multilumen central venous catheter (Cook, Bloomington, Ind) were placed. An $8 \mathrm{~F}$ coronary sinus catheter (Webster Lab Inc, Los Angeles, Calif) was introduced by fluoroscopic guidance into the great cardiac vein (GCV).

For 9 animals, a midline sternotomy was performed with careful sternal retraction, followed by sharp pericardotomy. The pleural cavity was not entered. Two microdialysis catheters (CMA/20 14/10 PC; CMA, Solna, Sweden) with names MD-A and MD-B were placed in the left ventricular wall, between the diagonal branches of the left anterior descending artery (LAD), below the midpoint of the LAD. A third microdialysis catheter, MD-C, was placed in the left ventricular wall in an area supplied by the circumflex artery as an internal control and backup sampling for microdialysis measurements. The catheters hubs were secured to the epicardium with a suture. The microdialysis polycarbonate membrane (outer diameter, $0.5 \mathrm{~mm}$ ) length was $10 \mathrm{~mm}$, and the membrane molecular weight cutoff was $20000 \mathrm{~d}$. Ringer's solution was pumped by syringe pumps (CMA 102, CMA) at a flow rate of $2 \mu \mathrm{L} / \mathrm{min}$. Dialysate was collected in individual tubes at 10-minute intervals. A miniaturized Clark electrode sensor (outer diameter, 0.46-0.6 mm; C1.2, Licox MCB; GMS, Kiel-Mielkendorf, Germany) for oxygen partial pressure in tissue (myocardial tissue oxygen tension $\left[\mathrm{PO}_{2}\right.$ tissue]) was placed and secured in the anterior left ventricular wall near the apex. The LICOX temperature probe was placed in the pericardium for temperature calibration of signal (LICOX CMP Tissue Oxygen Pressure Monitor, GMS).

\subsection{Pacing}

Two epicardial pacing wires (Flexon 2-0; US Surgical/ Syneture, Norwalk, Conn) were sewn to the surface of the right atrial appendage. Pacing at predetermined HRs was accomplished using the atrial pacing wires and an external pace generator (Pacesetter 3077; Osypka, Rheinfeld-Herten, Germany) in the VVI mode. After placement of catheters and pacing wires, the sternotomy was closed using sutures.

\subsection{Measurements}

Arterial and coronary venous (GCV) blood samples were drawn simultaneously for myocardial blood lactate measurements at baseline and at 5 minutes after each stepwise increase of paced HR level. Whole blood lactate analyses were immediately performed in the experimental laboratory (Yellow Springs Lactate Analyzer 1500; Yellow Springs, Ohio). The CMA 600 Microdialysis Analyzer (CMA Microdialysis $\mathrm{AB}$ ) was used for assessment of glucose, lactate, and pyruvate in MD-A and MD-C samples for each measurement point in the protocol. MD-B samples were tested for $\left[\mathrm{K}^{+}\right]$. The samples were diluted with ion-filtered water from 20 to $5000 \mu \mathrm{L}$ (250 times) and analyzed by a certified commercial laboratory, which used a quantitative flame atomic absorption spectroscopy detection method (Perkin-Elmer Analyser 100; Boston, Mass). The laboratory's estimated variation in measurement was reported as $5 \%$ at detected levels of $4 \mathrm{mmol} / \mathrm{L}$.

\subsection{Continuous vectorcardiography}

For vectorcardiography, 8 external electrodes (silver skin electrodes; Hewlett-Packard, Palo Alto, Calif) were applied to the shaved skin over and around the precordium, according to the system described by Frank [9]. The hardware and software used for computerized vectorcardiography were the MIDA 1000 vectorcardiography system (Ortivus Medical $\mathrm{AB}$, Täby, Sweden), which has been used in several previous experimental and clinical studies [10-13]. The signals were sampled at $500 \mathrm{~Hz}$ and averaged for periods of either 20 or 30 seconds as 3 orthogonal components, which were projected along the 3 perpendicular axes $(\mathrm{x}, \mathrm{y}$, and $\mathrm{z})$. The ST-segment deviations were measured 20 milliseconds after the J point. The absolute variable, spatial ST vector 
magnitude (ST-VM), was calculated according to the formula: $\mathrm{ST}-\mathrm{VM}=\sqrt{;\left(x^{2}+y^{2}+z^{2}\right)} \mu \mathrm{V}$. The absolute vector direction or angle (ST-VA) in degrees with reference to the y (sagittal)-axis was recorded. The VCG ST-segment measurements from the second to the ninth minutes during the 10-minute pacing sequence were averaged to provide a single estimate of ST-VM for that experimental HR. The relative variable spatial ST change vector magnitude (STC-VM) or the vector distance from the initial ST vector to the current was calculated as follows: $\mathrm{STC}-\mathrm{VM}=$ $\sqrt{;\left(x_{i}-x_{0}\right)^{2}+\left(y_{i}-y_{0}\right)^{2}+\left(z_{i}-z_{0}\right)^{2}} \mu \mathrm{V}$, where $i$ stands for actual orthogonal lead measurement and 0 stands for baseline reference measurement. The change in direction of ST vector angle (ST change vector angle [STC-VA]) was calculated as the difference between the initial and current ST-VA.

Also, a 12-lead ECG (bandwidth, 0.05-160 Hz) recording was obtained during the ninth minutes of each measurement period on a 6-channel recorder (Siecard 460; Siemans-Elema, Stockholm, Sweden). The paper speed was $50 \mathrm{~mm} / \mathrm{s}$, and the calibration was $1 \mathrm{mV}=10 \mathrm{~mm}$. Changes by manual measurements were noted starting at $0.5 \mathrm{~mm}(0.05 \mathrm{mV})$ and higher, and were rounded to the nearest $0.5 \mathrm{~mm}$. A summation of ST-segment change from baseline in all 12 leads (except aVR) was performed for all measurements [14].

\subsection{Four-part protocol}

\subsubsection{Presternotomy and poststernotomy control, at baseline $H R$}

Before the sternotomy, a VCG measurement was made at a nonpaced resting HR $(<100$ beats per minute). Then, the VCG leads were disconnected, and the sternotomy and pericardiotomy were performed, including instrumentation as described previously. The sternotomy was closed loosely with sutures and electrodes were replaced in the same positions, and a second (poststernotomy) VCG measurement was recorded at resting nonpaced HR for comparison to the presternotomy VCG. After sternotomy, there was a rest period of 1 hour before pacing was started.

\subsubsection{Pacing}

A baseline VCG reference measurement was recorded at a lowest pacing level, which was either 85 or 100 beats per minute depending on the animal's underlying resting HR. Measurements were gathered at each paced HR for 10 minutes, and HR increases were made in steps of 15 beats per minute up to the point at which consistent pacing capture no longer was obtained.

\subsubsection{Regional ischemia}

After the pacing protocol was completed, a second instrumentation procedure took place where a balloon catheter was placed into the mid-LAD to transiently occlude coronary blood flow to the areas sampled by the MD-A and MD-B catheters. The measurement point preischemia was collected before the start of coronary artery catheterization. Left main coronary artery catheterization was accomplished through an introducer (8F; Cordis, Miami Lakes, Fla) placed in the left internal carotid artery using a Scimed 7F guide catheter (Boston Scientific, Natick, Mass). Through this introducer catheter, a percutaneous transluminal coronary angioplasty perfusion dilation catheter with a perfusion lumen through the balloon area (Surpass $20 \mathrm{~mm}$, Scimed; Boston Scientific) was placed over a guide wire, with the balloon portion in the mid-LAD region. The balloon was inflated for 10 minutes, at the end of which, the ischemia measurement was collected, which comprised the last measurement point for the animals.

\subsubsection{Pacing without sternotomy}

In a separate group of 4 animals (who were anesthetized the same way), a transvenous pacing wire was placed into the right atrium (Elecath 4F bipolar pacing catheter; Elecath, Rahway, NJ). A 7F thermistor-tipped coronary sinus catheter (CCS-7U-90B, ball end; Webster Lab Inc) was placed in the $\mathrm{GCV}$, and GCV flow was measured using the retrograde thermodilution technique, using a Wheatstone bridge (CBA210, Webster Lab Inc, Baldwin Park, CA) and a Gould amplifier (Gould TA 5000; Cleveland, Ohio). Great cardiac

Table 1

Hemodynamic and metabolic findings during atrial pacing

\begin{tabular}{|c|c|c|c|c|c|c|c|c|}
\hline & \multicolumn{7}{|c|}{ HR (beats per minute) } & \multirow[t]{2}{*}{$P$} \\
\hline & $85(n=6)$ & $100(\mathrm{n}=9)$ & $115(n=9)$ & $130(n=9)$ & $145(n=9)$ & $160(\mathrm{n}=9)$ & $175(n=9)$ & \\
\hline MAP (mm Hg) & $84 \pm 3.4$ & $86 \pm 4.0$ & $89 \pm 4.4$ & $88 \pm 4.9$ & $89 \pm 5.2$ & $85 \pm 5.4$ & $76 \pm 5.6$ & .002 \\
\hline CVP (mm Hg) & $8 \pm 1.0$ & $8 \pm 0.8$ & $7 \pm 0.9$ & $7 \pm 0.9$ & $7 \pm 0.9$ & $8 \pm 0.9$ & $9 \pm 1.0$ & .000 \\
\hline $\mathrm{PO}_{2}$ tissue $(\mathrm{mm} \mathrm{Hg})$ & $66.0 \pm 4.3$ & $58.3 \pm 5.5$ & $56.3 \pm 5.2$ & $54.0 \pm 5.4$ & $51.6 \pm 5.8$ & $48.6 \pm 6.2$ & $42.8 \pm 7.4$ & .000 \\
\hline 12-lead ECG $\Delta$ STsum (mm) & - & $0.4 \pm 0.3$ & $2.8 \pm 0.3$ & $3.0 \pm 0.4$ & $3.2 \pm 0.4$ & $3.5 \pm 0.6$ & $3.4 \pm 0.4$ & .000 \\
\hline \multicolumn{9}{|l|}{ Coronary lactate } \\
\hline Extraction (\%) & $50.6 \pm 3.0$ & $49.5 \pm 2.0$ & $44.5 \pm 3.4$ & $43.3 \pm 4.2$ & $44.2 \pm 3.2$ & $44.0 \pm 2.9$ & $40.8 \pm 4.0$ & .052 \\
\hline a-gcv diff (mmol) & $0.49 \pm 0.03$ & $0.53 \pm 0.04$ & $0.47 \pm 0.07$ & $0.43 \pm 0.06$ & $0.43 \pm 0.04$ & $0.42 \pm 0.04$ & $0.39 \pm 0.04$ & .052 \\
\hline \multicolumn{9}{|l|}{ Microdialysis } \\
\hline Lactate $(\mathrm{mmol} / \mathrm{L})$ & $0.47 \pm 0.05$ & $0.51 \pm 0.04$ & $0.51 \pm 0.04$ & $0.55 \pm 0.04$ & $0.55 \pm 0.05$ & $0.57 \pm 0.05$ & $0.60 \pm 0.06$ & .054 \\
\hline Glucose $(\mathrm{mmol} / \mathrm{L})$ & $2.01 \pm 0.07$ & $2.09 \pm 0.07$ & $2.08 \pm 0.08$ & $2.12 \pm 0.07$ & $2.08 \pm 0.07$ & $2.05 \pm 0.08$ & $1.91 \pm 0.08$ & .000 \\
\hline Potassium $(\mathrm{mmol} / \mathrm{L})$ & $3.99 \pm 0.12$ & $4.15 \pm 0.09$ & $4.01 \pm 0.08$ & $4.13 \pm 0.10$ & $4.02 \pm 0.08$ & $4.03 \pm 0.08$ & $4.13 \pm 0.07$ & .525 \\
\hline Lactate-pyruvate ratio & $12.08 \pm 3.6$ & $11.1 \pm 1.9$ & $10.2 \pm 1.8$ & $9.3 \pm 1.3$ & $8.6 \pm 1.2$ & $8.1 \pm 1.0$ & $8.1 \pm 1.0$ & .020 \\
\hline
\end{tabular}

MAP indicates mean arterial pressure; CVP, central venous pressure; a-gcv diff, arterial-great coronary venous difference in concentration. $\Delta$ STsum, the summation of ST segment change in all 12 leads (except a VR) from baseline paced HR. $P$ values are from repeated measures analysis of variance (100-175 beats per minute). 

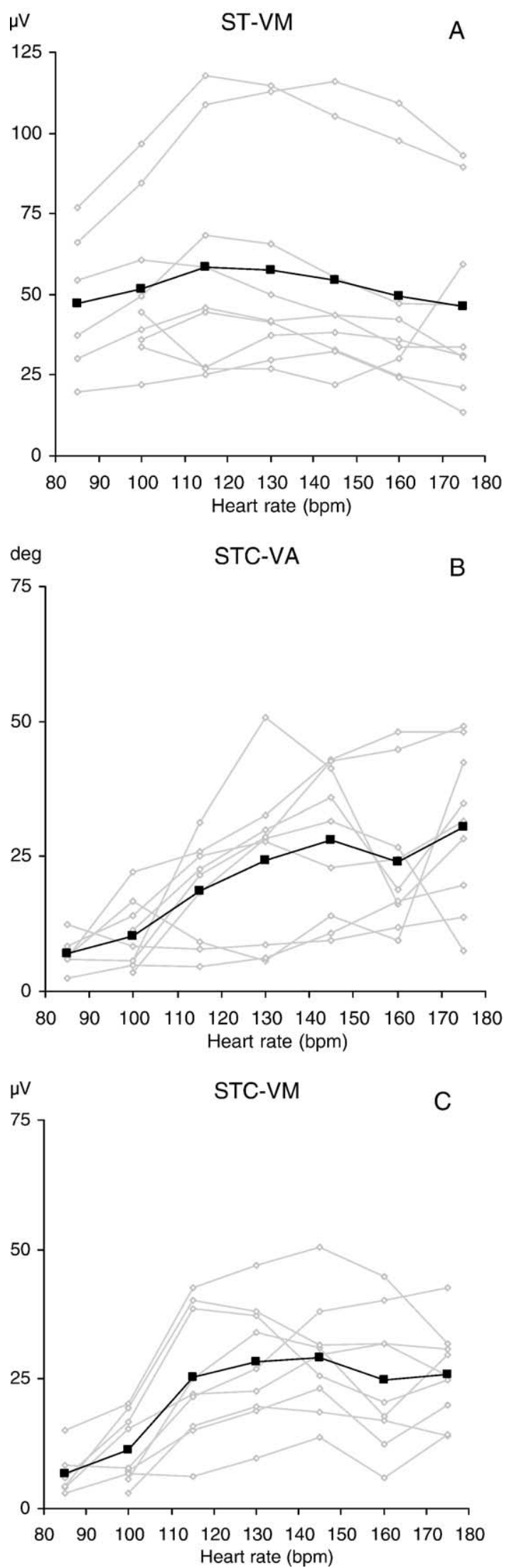

vein flow measurements were performed immediately after GCV (and arterial) blood samples were collected for myocardial oxygen consumption measurement. No sternotomy was performed in these 4 animals, which were put through the same HR and VCG protocol as described previously.

\subsection{Analysis and statistics}

Calculations of hemodynamic variables were made as follows: transcoronary myocardial lactate differences $=$ ([lactate $]_{\text {arterial }}-[\text { lactate }]_{g c v}$ ), and transcoronary lactate extraction percent $=\left([\text { lactate }]_{\text {arterial }}-[\text { lactate }]_{g c v}\right) /$ $[\text { lactate }]_{\text {arterial }} \times 100$ [15]. Further abbreviations and hemodynamic calculations are found hereinafter.

$$
\begin{aligned}
\mathrm{CaO}_{2}= & \left(\left(a \mathrm{HbO}_{2} / 100\right) \times a \mathrm{Hb} \times 1.39\right)+\left(\mathrm{PaO}_{2} \times 0.23\right) \\
\mathrm{CgcvO}_{2}= & \left(\left(g c v H b \mathrm{H}_{2} / 100\right) \times g c v H b \times 1.39\right) \\
& +\left(\mathrm{PgcvO}_{2} \times 0.23\right) \\
\mathrm{MVO}_{2}= & Q_{g c v} / 1000 \times\left(\mathrm{CaO}_{2}-\mathrm{CgcvO}_{2}\right)
\end{aligned}
$$

\section{Abbreviations}

$\mathrm{aHb} \quad$ Arterial hemoglobin $(\mathrm{g} / \mathrm{L})$

$\mathrm{aHbO}_{2} \quad$ Arterial saturation (\%)

$\mathrm{PaO}_{2} \quad$ Arterial $\mathrm{PO}_{2}(\mathrm{kPa})$

$\mathrm{CaO}_{2}$ Arterial oxygen content $(\mathrm{mL} / \mathrm{L})$

gcvHb Great cardiac venous hemoglobin $(\mathrm{g} / \mathrm{L})$

$\mathrm{gcvHbO}_{2}$ Great cardiac venous saturation (\%)

$\mathrm{PgcvO}_{2} \quad$ Great cardiac venous $\mathrm{PO}_{2}(\mathrm{kPa})$

$\mathrm{CgcvO}_{2}$ Great cardiac venous oxygen content $(\mathrm{mL} / \mathrm{L})$

$\mathrm{Q}_{\text {gcv }} \quad$ Great cardiac venous flow $(\mathrm{mL} / \mathrm{min})$

$\mathrm{MVO}_{2} \quad$ Myocardial oxygen consumption ( $\mathrm{mL} / \mathrm{min}$ )

ST-VM, STC-VA, and STC-VM values were grouped for absolute HRs (mean \pm SEM). Analyses were performed to detect changes in all parameters grouped for HR during the pacing protocol using repeated measurements analysis of variance test. Paired comparisons were made using the paired Student $t$ test. A $P$ value less than .05 was used to determine statistical significance. To describe variation within each pacing HR level for repeated STC-VM measurements each 15 seconds, an SD was calculated. Then, the average of these SDs was reported for different HR levels.

\section{Results}

Nine pigs (mean weight, $40 \pm 1 \mathrm{~kg}$ ) were atrially paced at a rate higher than their resting HR, starting either at

Fig. 1. In separate panels, ST-VM (A), STC-VA (B), and STC-VM (C) are shown for the course of pacing-induced HR increase. ST vector magnitude mean values are shown, and no systematic change was observed for the grouped values related to increases in HR. ST change vector magnitude increase during HR changes is shown, which is the main finding of the study. It is the combination of ST-VM and STC-VA that results in STCVM. This figure shows the relatively large contribution of STC-VA to STCVM results during HR increases. bpm indicates beats per minute. 
85 ( 6 animals) or at 100 beats per minute ( 3 animals). They were successfully paced at 15 -beats per minute increments up to 175 (all), 190 (4 animals), or 205 (2 animals) beats per minute. All animals were successfully paced between 100 and 175 beats per minute, and these results were analyzed further. Hemodynamic and metabolic findings are presented in Table 1. Mean arterial pressure and central venous pressure from resting (prepacing) did not differ from pacing baseline levels. The microdialysis results are reported for all 9 animals in the sternotomy group and measurement sequences at HRs 85 to 175 beats per minute (Table 1).

\subsection{Pacing and microdialysis measurements}

There were no increases in lactate or glucose during HR increases, which demonstrated that ischemic conditions were not present in the regions sampled by these probes. Pyruvate concentrations increased progressively with increasing HR. Lactate-pyruvate ratio decreased during HR increases. Transcoronary lactate concentration differences (arterial-great coronary venous difference) did not change during heart increases. These microdialysis and coronary blood measurements did not identify any metabolic findings of ischemia at any point in the pacing-induced $\mathrm{HR}$ increase sequences. A systematic relation of myocardial $\mathrm{PO}_{2}$ tissue to $\mathrm{HR}$ was observed, with $\mathrm{PO}_{2}$ tissue decreasing progressively during increasing HR, ranging from mean 66 to $43 \mathrm{~mm} \mathrm{Hg}$ (Table 1).
No animals at any measurement point had an arterial blood $\mathrm{PO}_{2}$ less than $140 \mathrm{~mm} \mathrm{Hg}$.

The mean $\left[\mathrm{K}^{+}\right]$as sampled by the myocardial microdialysis probe did not change over the course of HR increases, and the mean $\left[\mathrm{K}^{+}\right]$range in the microdialysate was from minimum $4.01 \pm 0.08$ to a maximum $4.15 \pm$ $0.27 \mathrm{mmol} / \mathrm{L}$ (Table 1).

\subsection{Vectorcardiography}

ST vector magnitude showed no systematic changes related to HR increases. ST vector magnitude started between 20 and $80 \mu \mathrm{V}$, and the course of ST-VM absolute levels during HR increases was highly variable for different animals (Fig. 1). Mean values for ST-VM during HR increases ranged from 47 to $58 \mu \mathrm{V}$, with no change during the course of pacing (mean ST-VM values at absolute HRs). ST change vector angle and STC-VM results are also presented in Fig. 1. Mean STC-VM increased during the course of pacing-induced $\mathrm{HR}$ increases, from a start pacing level mean of $6.3 \pm 1.3 \mu \mathrm{V}$ up to $26.0 \pm 3.1 \mu \mathrm{V}(P<.01$; range, $4-50 \mu \mathrm{V})$ at $\mathrm{HR} 175$ beats per minute. The angular difference appears to account for a larger part of the STCVM results than do the ST-VM events during increasing HR. The variation within each measurement sequence for STC-VM results (all pacing levels) is described by an SD that ranges from 2.15 to 2.84 (mean, 2.42) $\mu \mathrm{V}$. A

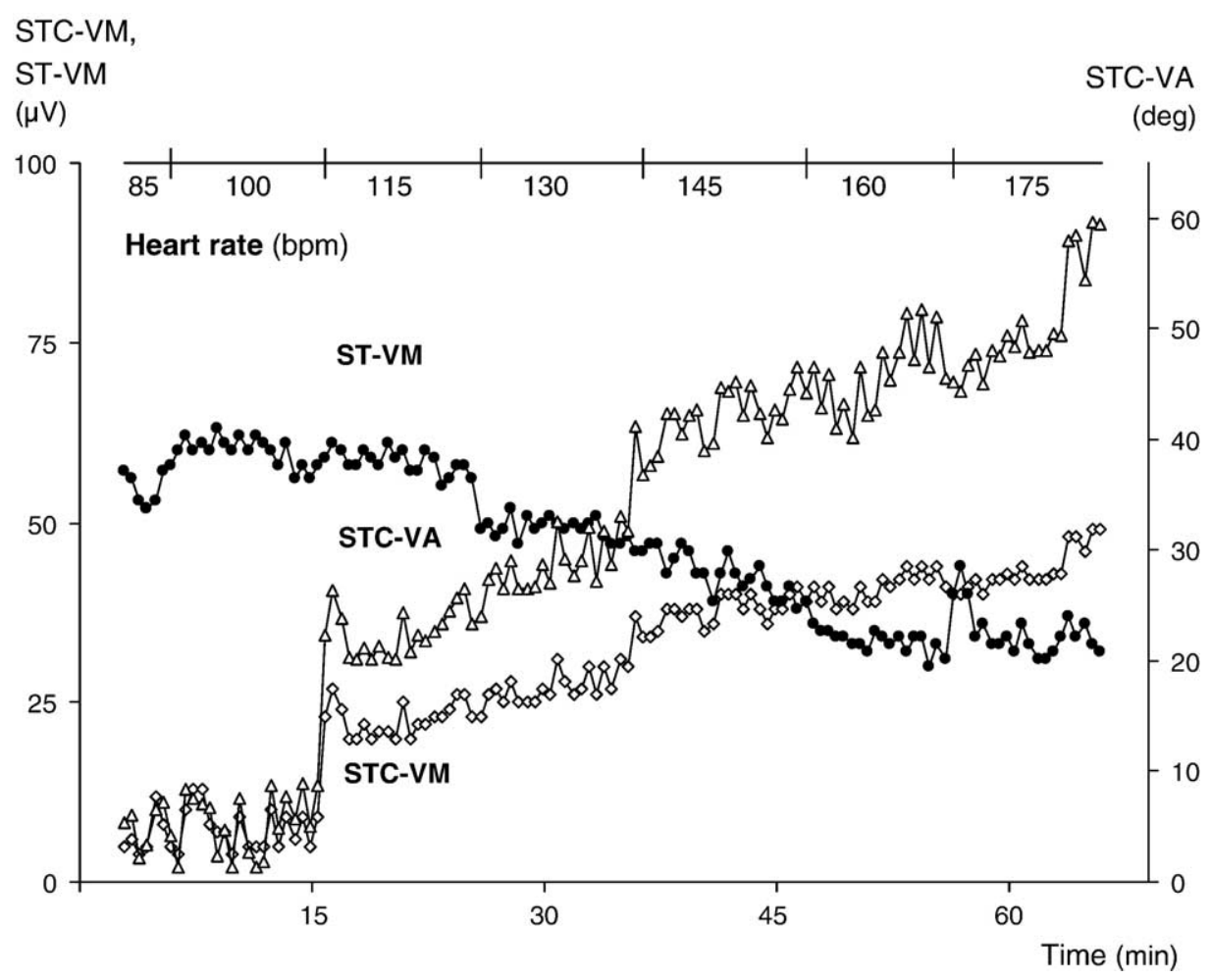

Fig. 2. This demonstrates representative raw data from a single animal for HR, ST-VM, STC-VA, and STC-VM during 10-minute pacing sequences. Each point represents the measurement from an averaged heart cycle during a 30 -second period. 
representative example of the pacing and measurement sequence is shown in Fig. 2.

\subsection{2-Lead ECG}

ST sum showed an increase associated with HR steps (Table 1), with much more marked changes during LAD occlusion (Table 2).

\subsection{Ischemic control (nonpacing)}

ST vector magnitude, along with STC-VA and STC-VM, responded to LAD occlusion with large significant increases, along with large increases in microdialysate lactate as well as myocardial lactate production detected by a change in transcoronary lactate difference (Table 2). Lactate-pyruvate ratio increased during LAD occlusion. Myocardial $\mathrm{PO}_{2}$ tissue decreased to very low levels in all animals during LAD occlusion. Microdialysate $\left[\mathrm{K}^{+}\right]$increased in all animals during LAD occlusion, from mean concentration $4.11 \pm$ 0.05 to $5.38 \pm 0.54 \mathrm{mmol} / \mathrm{L}$ (Table 2 ).

\subsection{Influence of the sternotomy, animals paced without sternotomy}

ST vector magnitude in 8 of 9 paced animals decreased in magnitude from presternotomy baseline to poststernotomy baseline, and the mean value decreased (Table 3). In 6 of 9, ST in the orthogonal lead Y (ST-Y) decreased from presternotomy to poststernotomy, whereas ST in the orthogonal lead Z (ST-Z), 9 of 9 increased. For the group of 4 animals with no sternotomy, pacing was conducted successfully at the same levels, from 85 to 175 beats per minute. For this group, STC-VM at HR 85 beats per minute started at mean $5.3 \pm 0.3 \mu \mathrm{V}$, with incremental progressive increases up to a mean of $31.6 \pm 8.3 \mu \mathrm{V}$ at HR 175 beats per minute. The STC-VM range for all these animals at HR 85 to 175 beats per minute was 5 to $51 \mu \mathrm{V}$. ST change vector angle

Table 2

Hemodynamic, metabolic, and VCG data before and after 10 minutes of LAD occlusion

\begin{tabular}{lcrr}
\hline & Preischemia & \multicolumn{1}{l}{ Ischemia } & $P$ \\
\hline HR (beats per minute) & $100 \pm 6$ & $128 \pm 10$ & .016 \\
MAP (mm Hg) & $71 \pm 2.3$ & $55 \pm 4.5$ & .007 \\
CVP (mm Hg) & $9 \pm 0.8$ & $10 \pm 0.7$ & .090 \\
PO $_{2}$ tissue $(\mathrm{mm} \mathrm{Hg})$ & $57.8 \pm 5.5$ & $8.2 \pm 6.1$ & $<.001$ \\
12-lead ECG $\Delta$ STsum $(\mathrm{mm})$ & - & $17.6 \pm 4.7$ & $<.001$ \\
Coronary lactate & & & \\
$\quad$ Extraction $(\%)$ & $45.6 \pm 3.9$ & $-108.1 \pm 28.8$ & $<.001$ \\
a-gcv diff (mmol) & $0.47 \pm 0.05$ & $-2.36 \pm 1.35$ & $<.001$ \\
Microdialysis & & & \\
Lactate (mmol/L) & $0.78 \pm 0.16$ & $3.14 \pm 1.03$ & $<.001$ \\
Glucose (mmol/L) & $1.73 \pm 0.12$ & $0.48 \pm 0.16$ & $<.001$ \\
Potassium $(\mathrm{mmol} / \mathrm{L})$ & $4.11 \pm 0.05$ & $5.38 \pm 0.18$ & $<.001$ \\
Lactate-pyruvate ratio & $8.4 \pm 0.8$ & $156.0 \pm 50.7$ & $<.001$ \\
Vectorcardiography & & & \\
STC-VM $(\mu \mathrm{V})$ & $15 \pm 3$ & $592 \pm 95$ & $<.001$ \\
ST-VM $(\mu \mathrm{V})$ & $36 \pm 3$ & $600 \pm 96$ & $<.001$ \\
STC-VA $\left({ }^{\circ}\right)$ & $14 \pm 3$ & $106 \pm 12$ & $<.001$ \\
\hline
\end{tabular}

12-Lead ECG $\Delta$ STsum is change from preischemia measurement. $P$ values are from paired $t$ test.
Table 3

Influence of the sternotomy

\begin{tabular}{lccc}
\hline & Presternotomy & Poststernotomy & $P$ \\
\hline ST-VM & $63 \pm 4$ & $44 \pm 6$ & .047 \\
ST-X & $27 \pm 7$ & $37 \pm 8$ & .297 \\
ST-Y & $20 \pm 5$ & $4 \pm 6$ & .133 \\
ST-Z & $-48 \pm 4$ & $1 \pm 4$ & .000
\end{tabular}

Values are in microvolts. The 3 orthogonal components, $x, y$, and $z$, before and after sternotomy demonstrate changes after sternotomy, leading to STVM decreases $(n=9)$. The ST magnitudes for the 3 orthogonal components, X, Y, Z, are presented respectively as ST-X, ST-Y and ST-Z.

started at $4.7^{\circ} \pm 1.0^{\circ}$ at $\mathrm{HR} 85$ beats per minute and increased to $44.8^{\circ} \pm 24.5^{\circ}$ at 175 beats per minute. Myocardial oxygen consumption $\left(\mathrm{MVO}_{2}\right)$ in the 4 animals did not change during the course of increased HR, as assessed by the GCV flow and oxygen content measurements.

\section{Discussion}

The main finding of this study concerned STC-VM, which is one of the parameters routinely used in monitoring of myocardial ischemia when vectorcardiography is used. ST change vector magnitude progressively changed during pacing-induced increases in HR, and these elevations in STC-VM occurred without any signs of myocardial ischemia in careful metabolic analyses of local metabolic markers. This supports recent findings from a clinical study where VCG ST signals were analyzed during pacing-induced HR increases in patients without coronary artery disease and ST magnitude changes were observed at voltage ranges possibly suggestive of ischemia, although without corroborative metabolic findings [5]. ST vector magnitude is the other routinely used VCG parameter. The current study also confirmed the heterogeneous response of ST-VM to HR changes observed in the aforementioned clinical series, although STC-VA results were not presented there. ST change vector angle is not routinely used (in the coronary care unit). The study design here provided systematic and stepwise increases in HR without producing ischemia. Progressive changes in metabolic markers were observed during $\mathrm{HR}$ increase, with decreasing myocardial $\mathrm{PO}_{2}$ tissue along with increasing pyruvate production, although none of these findings were consistent with an ischemic mechanism for HR-related ST deviation.

The disparity of ST-VM and STC-VM results is clarified by demonstration of changes in the ST vector angle (STCVA). The analysis of angular change during an event and understanding of this direction shift as an equally large contributor to STC-VM as magnitude is one aspect of the strength and depth of VCG analysis. There is no clear explanation from these results for the STC-VA changes during increased HRs, but it is important to recognize that they exist and contribute markedly to STC-VM changes during $\mathrm{HR}$ alterations. Without this insight, there is a risk that ST-VM or STC-VM findings by themselves in individual patients can be interpreted incorrectly with regard to levels 
commonly associated with ischemia. Whenever STC-VM or ST-VM is presented, analysis of STC-VA may provide further information. In theory and in practice, ischemic events can occur where there are changes in angular direction but not in absolute ST-VM, which is the explanation for why STC-VM can detect ischemia despite ST-VM remaining unchanged [16]. The converse is unlikely, that ST-VM can change with no alteration in STC-VM.

The HR-related STC-VM increases correspond to previously published clinical observations in exercise testing of coronary care patients in which higher STC-VM were observed in relation to higher HRs $[3,4]$, although ischemia in those settings was not directly assessed by a reliable second method. Also, the response of STC-VM has not been previously studied in response to graded and controlled HR increases. The novelty of the main findings here is that this is the first study where HR-related ST vector changes have been documented in connection with definitive absence of myocardial ischemia. A measurement before and during a controlled regional ischemic event was included in our study design to verify the response in both the vectorcardiography and the microdialysis/biochemical detection of ischemia with a true-positive finding.

The STC-VM trend during HR increases supports suggestions that ST changes need to be either adjusted for HR, or otherwise, HR adjustments for ischemic thresholds need to be described and validated. Adjustment of ST-segment changes in the standard 12-lead ECG during exercise testing was proposed already more than 20 years ago to enhance the discrimination between myocardial ischemia and physiological ST changes during conditions of increased HR [17]. Although similar speculation about VCG ST analysis and HR relations has been published $[3,4]$, this is the first study to measure the course of VCG ST events during a course of graded HR increase with simultaneous metabolic local assessment of myocardium.

\subsection{Metabolic local myocardial conditions during increasing $H R$}

There were changes in the measured metabolic parameters during increased $\mathrm{HR}$, including $\mathrm{PO}_{2}$ tissue, glucose, pyruvate, and lactate-pyruvate ratio, although none of these observations were suggestive of ischemia or injury, as demonstrated in Table 2. The changes demonstrated in the microdialysis results probably do not reflect absolute concentrations but rather a sampling of a concentration change, which provides information about direction and general magnitude of change but not accurate tissue concentrations of substances. The reason for pyruvate increase during pacing and HR increases is unclear, and pyruvate increase of a similar magnitude and time frame has been reported previously [18] in the same type of model even during control (no increased HR condition) measurements. This suggests that pyruvate increases were not related to HR increases. Pyruvate concentration would be expected to decrease if ischemia was present. Lactatepyruvate ratio decreased during pacing in conjunction with increasing pyruvate and static lactate levels. During the ischemia measurement, there was a clear increase in this ratio. If there had been ischemia during the pacing protocol, this ratio should have increased. Glucose decreased slightly during pacing but showed another magnitude of decreases during ischemia. These metabolic findings during HR increase can be interpreted as consistent with myocardial work-related changes in local interstitial concentrations of oxygen, glucose, and pyruvate, which occurred within a range or reserve for nonresting but nonischemic myocardial conditions during pacing. In the group of 4 animals with no sternotomy, where $\mathrm{MVO}_{2}$ was measured, there was no indication of a large HR-related change in $\mathrm{MVO}_{2}$ during pacing, but this was not tested in the sternotomy animals because of lack of availability of a flow-measuring coronary sinus catheter at the time of those experiments.

One possible mechanism of nonischemic ST level changes during HR increases may be alterations in extracellular $\left[\mathrm{K}^{+}\right]$related to shorter intervals for potassium reuptake during repolarization, although the microdialysis sampling in our study did not demonstrate signs of change in myocardial extracellular $\left[\mathrm{K}^{+}\right]$during STC-VM increases and increased HRs. Extracellular $\left[\mathrm{K}^{+}\right]$changes during HR increases have been demonstrated experimentally in atrial muscle [19] but not in ventricular muscle. Also, microdialysis sampling to assess HR-related changes in extracellular $\left[\mathrm{K}^{+}\right]$provides a time-averaged single concentration, which may not reflect very rapid or dynamic ion concentration changes. A fast-response $\mathrm{K}^{+}$electrode placed in the myocardium would be required to increase the temporal resolution of $\mathrm{K}^{+}$measurements.

The findings in the nonsternotomized animals did not suggest another pattern for ST change related to HR, although in that small group of animals, the mean STC-VM values were slightly higher at the highest HR than those from the sternotomized animals, although there was not sufficient power in the results to provide a definitive test between sternotomy and nonsternotomy. The sternotomy and pericardotomy preparation by itself leads to a change in orthogonal lead voltages (and ST-VM), most likely because of a change in heart position in the chest after the pericardium is opened widely, with the heart apex falling more posterior in with the animals in the supine position. After analyzing the results for 4 nonsternotomized animals, with no apparent large STC-VM differences related to HR compared with the other 9 pigs, we determined that further use of laboratory animals in that subseries was unwarranted.

There are several different conditions, other than ischemia, that can cause ST changes, and HR elevation can be associated with these factors in clinical situations. A theoretical but unlikely mechanism of STC-VM increase at higher HRs is the possible effect of atrial repolarization if it overlaps onto the ST phase. Previous direct measurement and analysis of this [20] have suggested that atrial repolarization forces are unlikely to affect STC-VM changes in relation to increasing HR. 


\subsection{Methodology for VCG ST-VM measurement}

Technical considerations concerning measurement of ST-VM can affect the results. We chose to present the average ST-VM for the period 2 to 9 minutes to minimize artifacts. The variation during measurement sequences was quite small (SD, $<2.5 \mu \mathrm{V})$, demonstrating the stability of the VCG ST segment during each HR step. This stability in ST segments for each HR supports the interpretation that ST findings were related to HRs specifically and not to some continuous and progressive metabolic process.

\subsection{Limitations of the model}

First, pacing-induced tachycardia as a model may have limitations in producing conditions that are similar to those in patients with an elevated HR where ischemia may be possible or suspected, and therefore, there may not be a strong clinical correlate for isolated tachycardia interventions in patients with coronary artery disease. Second, the magnitude of STC-VM responses to HR in pigs may not directly reflect the magnitude of similar type of response in humans. The study was designed to investigate the biologic phenomenon of STC-VM changes related to HR but not to calibrate normal or pathological STC-VM ranges for humans. Further clinical studies are needed to validate either a HR-related VCG ST magnitude adjustment or redefinition of clinical ischemic thresholds with reference to HR. Finally, the 12-lead ECG method, which we used here, was a crude monitoring system only and was not advanced enough to allow an in-depth evaluation of 12-lead ECG ST response to HR changes. Therefore, no findings here allow testing of the agreement of similar vectorcardiography and 12-lead ECG ST analysis systems for these HR-related ST changes.

\subsection{Conclusions}

Heart rate increases have effects on ST vector parameters that are not related to myocardial ischemia. Changes in STC-VM and ST-VM during increased HR need to be analyzed together with changes in vector direction/angle (STC-VA). These findings shed no new light on mechanism of ST changes during increasing HR. These HR effects need to be further analyzed in clinical conditions to improve the diagnostic specificity of vectorcardiography for myocardial ischemia in patients with changes in HR.

\section{Acknowledgments}

This study was supported by grants from the Heart Foundation of Northern Sweden; Umeå, Sweden; Swedish Medical Research Council; Stockholm, Sweden; Swedish Heart-Lung Fund; Stockholm, Sweden and Umeå University, Umeå, Sweden. The authors acknowledge the valuable assistance from Anna-Maja Åberg and Pernilla Abrahamsson.

\section{References}

[1] Maroko PR, Libby P, Covell JW, Sobel BE, Ross J, Braunwald E. Precordial S-T segment elevation mapping: an atraumatic method for assessing alterations in the extent of myocardial ischemic injury. The effects of pharmacologic and hemodynamic interventions. Am J Cardiol 1972;29:223.

[2] Menown IB, Mackenzie G, Adgey AA. Optimizing the initial 12-lead electrocardiographic diagnosis of acute myocardial infarction. Eur Heart J 2000;21:275.

[3] Pilhall M, Riha M, Jern S. Ischaemic heart disease and the changes in the QRS and ST segments during exercise: a pilot study with a novel vectorcardiographic system. Clin Physiol 1992;12:209.

[4] Jensen SM, Häggmark S, Johansson G, Näslund U. On-line computerized vectorcardiography: influence of body position, heart rate, radiographic contrast fluid and myocardial ischemia. Cardiology 1997; $88: 576$

[5] Häggmark S, Haney MF, Jensen SM, Johansson G, Näslund U. ST-segment deviations during pacing-induced increased heart rate in patients without coronary artery disease. Clin Physiol Funct Imaging 2005;25:246.

[6] Heller GV, Aroesty JM, McKay RG, et al. The pacing stress test: a reexamination of the relation between coronary artery disease and pacing-induced electrocardiographic changes. Am J Cardiol 1984; 54:50.

[7] Okin PM, Grandits G, Rautaharju PM, et al. Prognostic value of heart rate adjustment of exercise-induced ST segment depression in the multiple risk factor intervention trial. J Am Coll Cardiol 1996; 27:1437.

[8] National Research Council. Guide for care and use of laboratory animals. 7th ed. National Academy of Science. Washington, DC, USA: National Academy Press; 1996.

[9] Frank E. Accurate, clinically practical system for spatial vectorcardiography. Circulation 1956;13:737.

[10] Näslund U, Häggmark S, Johansson G, Reiz S. Quantification of myocardium at risk and detection of reperfusion by dynamic vectorcardiographic ST segment monitoring in a pig occlusion-reperfusion model. Cardiovasc Res 1993;27:2170.

[11] Dellborg M, Svensson AM, Johansson M, Swedberg K. Early electrocardiographic changes in acute myocardial infarction treated by streptokinase or alteplase: a randomized study with dynamic, multilead, electrocardiographic monitoring. Cardiology 1993;82:368.

[12] Nørgaard BL, Rasmussen BM, Dellborg M, Thygesen K. Temporal and positional variability of the ST segment during continuous vectorcardiography monitoring in healthy subjects. J Electrocardiol 1999; 32:149.

[13] Jensen SM, Karp K, Rask P, Näslund U. Assessment of myocardium at risk with computerized vectorcardiography and technetium-99msestamibi-single photon emission computed tomography during coronary angioplasty. Scand Cardiovasc J 2002;36:11.

[14] Gwechenberger M, Schreiber W, Kittler H, et al. Prediction of early complications in patients with acute myocardial infarction by calculation of the ST score. Ann Emerg Med 1997;30:563.

[15] Gonschior P, Gonschior GM, Conzen PF, et al. Myocardial oxygenation and transmural lactate metabolism during experimental acute coronary stenosis in pigs. Basic Res Cardiol 1992;87:27.

[16] Jensen SM, Johansson G, Osterman G, Reiz S, Näslund U. On-line computerized vectorcardiography monitoring of myocardial ischemia during coronary angioplasty: comparison with 12-lead electrocardiography. Coron Artery Dis 1994;5:507.

[17] Elamin MS, Boyle R, Kardash MM, et al. Accurate detection of coronary heart disease by new exercise test. Br Heart J 1982;48:311.

[18] Wikström G, Ronquist G, Nilsson S, Maripu E, Waldenström A. Continuous monitoring of energy metabolites using microdialysis during myocardial ischaemia in the pig. Eur Heart J 1995;16:339.

[19] Miyata A, Dowell JD, Zipes DP, Rubart M. Rate-dependent $[\mathrm{K}+]_{\mathrm{o}}$ accumulation in canine right atria in vivo: electrophysiological consequences. Am J Physiol Heart Circ Physiol 2002;283:H506.

[20] Debbas NM, Jackson SH, de Jonghe D, Robert A, Camm AJ. Human atrial repolarization: effects of sinus rate, pacing and drugs on the surface electrocardiogram. J Am Coll Cardiol 1999;33:358. 